\title{
Quinone Compound
}

National Cancer Institute

\section{Source}

National Cancer Institute. Quinone Compound. NCI Thesaurus. Code C796.

A class of organic compounds with the base structure of quinone, an aromatic benzene molecule containing a double ketone functional group. 